Proteção de Plantas

(ConTrole Químico, ResistênCIA de Plantas E MANEJo

INTEGRADO DE PRAGAS)

\title{
Controle Químico e Mecânico de Cupins de Montículo (Isoptera: Termitidae) em Pastagens
}

\author{
José R. Valério ${ }^{1}$, Adão V. Santos ${ }^{1}$, Antônio P. Souza ${ }^{1}$, Cláudio A.M. Maciel ${ }^{1}$ \\ e Marlene C.M. Oliveira ${ }^{2}$ \\ ${ }^{1}$ EMBRAPA/CNPGC, Caixa postal 154, 79002-970, Campo Grande, MS. \\ ${ }^{2}$ EMPAER/MS, Caixa postal 51, 79114-000, Campo Grande, MS.
}

An. Soc. Entomol. Brasil 27(1): 125-131 (1998)

\section{Chemical and Mechanical Control of Mound-Building Termite Species (Isoptera: Termitidae) in Pastures}

\begin{abstract}
Studies were conducted aiming the control of mound-building termite species in pastures. Six insecticides were tested against the termite Cornitermes cumulans (Kollar) being the active ingredients (a.i.) and respective dosages (quantity of a.i. per termite mound) as follows: A - sulfluramid (ant granular bait $0,30 \%$ ): $A_{1}=60 \mathrm{mg}, A_{2}=120 \mathrm{mg}$ and $A_{3}=180 \mathrm{mg} ; B$ - chlorpyrifos (ant granular bait $0,125 \%$ ): $\mathrm{B}_{1}=25 \mathrm{mg}, \mathrm{B}_{2}=50 \mathrm{mg}$ and $\mathrm{B}_{3}=75 \mathrm{mg} ; \mathrm{C}$ - dodecachlor (ant granular bait 0,45\%): 90mg; D - abamectin (EC 1,8\%): 3,6 mg; E deltamethrin (DP 0,2\%): $\mathrm{E}_{1}=10 \mathrm{mg}$ and $\mathrm{E}_{2}=20 \mathrm{mg} ; \mathrm{F}$ - fipronil $(\mathrm{G} \mathrm{2 \%}): \mathrm{F}_{1}=25$ $\mathrm{mg}, \mathrm{F}_{2}=50 \mathrm{mg}$ and $\mathrm{F}_{3}=100 \mathrm{mg}$. Thirty days after insecticide application percent mortalities were: $A_{1}, A_{2}, B_{1}, E_{2}=0 ; A_{3}, B_{2}, B_{3}, E_{1}=10 \% ; C=90 \% ; D, F_{1}, F_{2}$, $\mathrm{F}_{3}=100 \%$. In a 2nd test, the insecticide fipronil was tested against three species of mound building termites, $C$. cumulans, $C$. bequaerti (Emerson) and Syntermes $\mathrm{sp}$, at $100 \mathrm{mg}$ of a.i./mound in the case of the first two species, or per $\mathrm{m}^{2}$ of mound, for Syntermes sp. The percent mortalities after 30 days were: $100 \%$ for C. cumulans and C. bequaerti and $50 \%$ for Syntermes sp. In a 3rd test, mechanical and chemical control were compared against $C$. cumulans. Mechanical control was performed using a special drill (which operates attached to a tractor) that completely destroy the termite mound. For the chemical treatment, the insecticide abamectin (EC 1,8\%; 3,6 mg a.i./mound) was utilized. Both treatments provided 90 to $100 \%$ control.
\end{abstract}

KEY WORDS: Insecta, chemical control, mechanical control, Cornitermes, Syntermes.

RESUMO - Conduziram-se três ensaios objetivando o controle de cupins de montículo em pastagens. No primeiro, testaram-se seis inseticidas no controle de Cornitermes cumulans (Kollar), sendo que os ingredientes ativos (i.a.) e respectivas doses (quantidade do i.a. por cupinzeiro) foram: A - sulfluramida (isca formicida 0,30\%): $\mathrm{A}_{1}=60 \mathrm{mg}, \mathrm{A}_{2}=120 \mathrm{mg}$ e $\mathrm{A}_{3}=180 \mathrm{mg}$; $\mathrm{B}$ - clorpirifós (isca formicida $0,125 \%$ ): $\mathrm{B}_{1}=25 \mathrm{mg}, \mathrm{B}_{2}=50 \mathrm{mg} \mathrm{e} \mathrm{B}_{3}=75 \mathrm{mg} ; \mathrm{C}$ - dodecacloro (isca formicida 0,45\%): $90 \mathrm{mg}$; D - abamectina (concentrado emulsionável 1,8\%): 
3,6 mg; E - deltametrina (pó seco 0,2\%): $\mathrm{E}_{1}=10 \mathrm{mg}$ e $\mathrm{E}_{2}=20 \mathrm{mg}$; F - fipronil (granulado 2\%): $\mathrm{F}_{1}=25 \mathrm{mg}, \mathrm{F}_{2}=50 \mathrm{mg} \mathrm{e} \mathrm{F}_{3}=100 \mathrm{mg}$. Os percentuais de mortalidade 30 dias após a aplicação foram: $\mathrm{A}_{1}, \mathrm{~A}_{2}, \mathrm{~B}_{1}, \mathrm{E}_{2}=0 ; \mathrm{A}_{3}, \mathrm{~B}_{2}, \mathrm{~B}_{3}, \mathrm{E}_{1}=10 \%$; $\mathrm{C}=90 \% ; \mathrm{D}, \mathrm{F}_{1}, \mathrm{~F}_{2}, \mathrm{~F}_{3}=100 \%$. No $2^{\circ}$ ensaio testou-se o produto fipronil no controle de $C$. cumulans, C. bequaerti (Emerson) e Syntermes sp, utilizando-se $100 \mathrm{mg}$ do i.a/cupinzeiro, no caso das duas primeiras espécies, e, por $\mathrm{m}^{2}$ de cupinzeiro no caso de Syntermes sp. Os percentuais de mortalidade 30 dias após a aplicação do produto foram: $100 \%$ para C. cumulans e C. bequaerti e $50 \%$ para Syntermes sp. No $3^{\circ}$ ensaio, avaliou-se a eficiência de método mecânico, através do implemento denominado "broca cupinzeira" no controle do C. cumulans. Houve dois tratamentos: destruição mecânica exclusivamente, e, tratamento químico (abamectina CE 1,8\%; 3,6 mg i.a. por cupinzeiro) associado à destruição mecânica. Neste ensaio, que foi conduzido em dois locais, constataram-se 90 a $100 \%$ de mortalidade.

PALAVRAS-CHAVE: Insecta, controle químico, controle mecânico, Cornitermes, Syntermes.

Os insetos-pragas associados às pastagens têm sido pouco estudados, especialmente em relação às diferentes espécies de cupins. A espécie Cornitermes cumulans (Kollar), comumente encontrada nas pastagens cultivadas das regiões Sudeste e Centro-Oeste do Brasil, no entanto, constitui uma exceção, principalmente no que tange à medidas de controle.

Se, de um lado, altas infestações de cupinzeiros dessa espécie são encontradas em pastagens, de outro não está claro se esse inseto estaria causando danos diretos às pastagens. $O$ único trabalho na tentativa de se avaliar os possíveis danos causados por esse inseto às pastagens foi conduzido por Cosenza \& Carvalho (1974). Estes autores concluíram, após observações conduzidas por 16 meses, que a eliminação do cupim de montículo (densidade média de 170 cupinzeiros por hectare) não alterou a produção de matéria seca, qualidade da pastagem, bem como a cobertura vegetal. Entre os possíveis danos indiretos atribuídos a C. cumulans, menciona-se a redução de área útil das pastagens, o que é questionável. Cosenza \& Carvalho (1974) afirmaram que a área total ocupada pelos cupinzeiros em pastagens não era significativa, sendo menor do que se supunha. Valério (1994) refere-se à área média ocupada por cupinzeiro como sendo ao redor de $0,5 \mathrm{~m}^{2}$ em amostragens feitas no Estado de Mato Grosso do Sul. Dados semelhantes foram encontrados por Holt \& Coventry (1982), na Austrália, para Amitermes vitiosus Hill, onde 286 cupinzeiros/ha com altura média de $54 \mathrm{~cm} \mathrm{e}$ diâmetro médio na base de $50 \mathrm{~cm}$, ocupavam apenas $0,6 \%$ da área.

Como danos indiretos os cupinzeiros podem abrigar animais peçonhentos, dificultar a movimentação de máquinas e animais, e depreciar a propriedade, conferindo-lhe um aspecto de abandono.

$\mathrm{O}$ controle tem sido feito predominantemente através do uso de inseticidas químicos. Até 1985, os inseticidas clorados eram os mais usados, mas devido a sua proibição, inseticidas alternativos passaram a ser testados (Motta et al. 1987, Mariconi et al. 1990, Valério et al. 1994). Testaram-se fungos entomopatógenos (Fernandes \& Alves 1991) e, mesmo método de controle alternativo, através da destruição mecânica (Ávila \& Rumiatto 1995). Procurouse adicionar informações sobre a eficiência de alguns produtos inseticidas, um deles testado em diferentes espécies de cupins de 
montículo, assim como de método mecânico.

\section{Material e Métodos}

Conduziram-se três ensaios, sendo que no $1^{\circ}$ (ensaio I) avaliaram-se diferentes inseticidas no controle de C. cumulans; no $2^{\circ}$ (ensaio II) avaliou-se um único inseticida no controle de C. cumulans, C. bequaerti e Syntermes sp., e, no $3^{\circ}$ (ensaio III), avaliouse a eficiência da destruição mecânica, através do implemento denominado "brocacupinzeira" no controle de C. cumulans.

Na véspera da aplicação dos produtos, os cupinzeiros de $C$. cumulans e $C$. bequaerti foram identificados e perfurados verticalmente até a câmara celulósica. Apenas os cupinzeiros cujos orifícios haviam sido tampados pelos cupins foram considerados vivos e, portanto, utilizados nos testes. Para o caso da espécie Syntermes sp., em cujos cupinzeiros não há uma câmara celulósica à semelhança das outras duas espécies, fez-se um corte na superfície dos cupinzeiros para confirmar se estavam vivos, com base na constatação de atividade das diferentes castas. A perfuração para aplicação do produto, neste caso, consistiu na penetração de uma barra de ferro através da camada do solo exposto, até uns 20 centímetros abaixo do nível do solo. Considerando que o cupinzeiro desta espécie pode ocupar área, às vezes, de vários metros quadrados, fêz-se, quando aplicável, uma perfuração por $\mathrm{m}^{2}$ de cupinzeiro. No ensaio I, com cupinzeiros de $50 \mathrm{~cm}$ de altura em média, foram avaliados os seguintes produtos e doses: A - Isca granulada com $0,3 \%$ de sulfluramida (doses: A1 $=60 \mathrm{mg}, \mathrm{A} 2=120 \mathrm{mg}$ e A3=180 mg i.a./cupinzeiro); B - Isca granulada com $0,125 \%$ de clorpirifós (doses: $\mathrm{B} 1=25 \mathrm{mg}, \mathrm{B} 2=50 \mathrm{mg}$ e B3=75 mg i.a./ cupinzeiro); C - Isca granulada com $0,45 \%$ de dodecacloro (tratamento padrão) (90 mg i.a./cupinzeiro); D - Concentrado emulsionável com $1,8 \%$ de abamectina (tratamento padrão) (3,6 mg i.a./0,5 l de água/cupinzeiro); E - Pó seco com $0,2 \%$ de deltametrina (doses: $\mathrm{E} 1=10 \mathrm{mg}$ e E2=20 mg i.a./cupinzeiro) e F Granulado com $2 \%$ de fipronil (doses: $\mathrm{F} 1=25$ mg, F2=50 mg e F3=100 mg i.a./cupinzeiro), totalizando treze tratamentos. Houve 10 repetições para cada tratamento, sendo cada cupinzeiro uma repetição. As avaliações foram feitas 30 dias após a aplicação dos produtos, quando todos os cupinzeiros foram destruídos e examinados quanto à presença de sobreviventes. Encontrando-se indivíduos vivos, mesmo que em reduzida quantidade, o cupinzeiro foi considerado vivo.

No ensaio II avaliou-se o inseticida fipronil, na formulação granulada com $2 \%$ de i.a., no controle de colônias de C. cumulans, C. bequaerti e Syntermes sp. Utilizou-se a dose de $100 \mathrm{mg}$ i.a. por cupinzeiro, sendo que, para a espécie Syntermes sp., esta dose foi aplicada por $\mathrm{m}^{2}$ do cupinzeiro, conforme mencionado anteriormente. A altura média dos cupinzeiros de C. cumulans e $C$. bequaerti era de $40 \mathrm{~cm}$, enquanto que a área média dos cupinzeiros de Syntermes sp. era de 1,8 $\mathrm{m}^{2}$, variando de 1 até $4 \mathrm{~m}^{2}$. Houve 10 repetições para cada tratamento e a avaliação foi feita à semelhança do mencionado para o $1^{\circ}$ ensaio.

No ensaio III avaliou-se a eficiência da "broca cupinzeira", um implemento que é acoplado ao diferencial de um perfurador de solo e acionado pela tomada de força do trator, comparando-se a mortalidade de cupinzeiros de $C$. cumulans apenas destruídos pela ação mecânica do implemento, com a de outros que, 30 dias antes de serem destruídos com este implemento foram tratados com o inseticida abamectina (3,6 mg i.a./0,5 1 de água/cupinzeiro). Para garantir completa destruição dos cupinzeiros com a broca, houve casos onde foram feitos mais do que uma perfuração no mesmo cupinzeiro, além de ter sido necessário contar com uma pessoa apoiada sobre o implemento para completa penetração da broca no solo, que na ocasião encontrava-se seco. Este ensaio, com seus dois tratamentos, foi instalado em dois locais (locais 1 e 2) dentro da fazenda experimental do Centro Nacional de Pesquisa de Gado de Corte (CNPGC), da Empresa Brasileira de Pesquisa Agropecuária (EMBRAPA). No local 1, onde utilizaram-se 36 cupinzeiros (18 repetições para cada tratamento), a altura 
média dos mesmos era de $47,5 \mathrm{~cm}$, enquanto que no local 2, onde utilizaram-se 38 cupinzeiros, sendo metade para cada tratamento, a média de altura era de $41 \mathrm{~cm}$. As avaliações foram conduzidas 70 dias após a aplicação do inseticida (40 dias após a destruição mecânica), quando os cupinzeiros foram examinados quanto à possível reconstrução dos ninhos. O cupinzeiro foi considerado morto quando não se constatou tal reconstrução.

\section{Resultados e Discussão}

Ensaio I. Os maiores percentuais de mortalidade foram conseguidos com dodecacloro, abamectina e com as três doses testadas de fipronil, sendo estes, respectivamente, $90,100,100,100$ e $100 \%$ (Tabela 1). Deltametrina e as iscas formicidas, uma à base de sulfluramida e a outra de clorpirifós, não exerceram controle eficiente, verificando-se mortalidade de no máximo 10\%. A alta eficiência de dodecacloro, abamectina e fipronil estão em concordância com resultados obtidos em outros trabalhos. Motta et al. (1987) e Buainain-Alves et al. (1993) relataram mortalidades acima de $90 \%$ com dodecacloro. Mariconi et al. (1994) e Valério et al. (1994) constataram, igualmente, alta eficiência com abamectina. Em função disso, esses dois ingredientes ativos foram utilizados como tratamentos padrões ou testemunhas. Mariconi et al. (1994) registraram controle eficiente com fipronil com doses variando de 300 a $400 \mathrm{mg}$ de ingrediente ativo por cupinzeiro. Ressalta-se que, $100 \%$ de mortalidade foi constatada com dose bem inferior, de apenas $25 \mathrm{mg}$ por cupinzeiro.

A isca formicida com sulfluramida, que substituiu no mercado a isca à base de

Tabela 1. Eficiência de alguns inseticidas no controle do cupim de montículo Cornitermes cumulans, aplicados na câmara celulósica, através de perfuração vertical e avaliados 30 dias após a aplicação.

\begin{tabular}{|c|c|c|c|c|c|}
\hline \multirow{2}{*}{$\begin{array}{l}\text { Tratamento } \\
\text { (i.a.) }\end{array}$} & \multirow[b]{2}{*}{ Formulação } & \multirow{2}{*}{$\begin{array}{c}\text { Concentração } \\
\text { i.a. }(\%)\end{array}$} & \multicolumn{2}{|l|}{ Dose } & \multirow{2}{*}{$\begin{array}{c}\text { Mortalidade } \\
\qquad(\%)\end{array}$} \\
\hline & & & $\begin{array}{l}\text { Produto } \\
\text { com./cup. }\end{array}$ & $\begin{array}{l}\text { i.a. } \\
\text { mg/cup. }\end{array}$ & \\
\hline$\overline{A_{1}-\text { Sulfluramida }}$ & isca granulada & 0,30 & $20 \mathrm{~g}$ & 60 & 0 \\
\hline $\mathrm{A}_{2}$-Sulfluramida & isca granulada & 0,30 & $40 \mathrm{~g}$ & 120 & 0 \\
\hline $\mathrm{A}_{3}^{2}$-Sulfluramida & isca granulada & 0,30 & $60 \mathrm{~g}$ & 180 & 10 \\
\hline B-Clorpirifós & isca granulada & 0,125 & $20 \mathrm{~g}$ & 25 & 0 \\
\hline $\mathrm{B}_{2}$-Clorpirifós & isca granulada & 0,125 & $40 \mathrm{~g}$ & 50 & 10 \\
\hline $\mathrm{B}_{3}^{2}$-Clorpirifós & isca granulada & 0,125 & $60 \mathrm{~g}$ & 75 & 10 \\
\hline $\mathrm{C}^{3}$ - Dodecacloro ${ }^{1}$ & isca granulada & 0,45 & $20 \mathrm{~g}$ & 90 & 90 \\
\hline D - Abamectina ${ }^{1}$ & C.E. ${ }^{2}$ & 1,8 & $0,2 \mathrm{ml}^{4}$ & 3,6 & 100 \\
\hline$E_{1}$-Deltametrina & P.S. ${ }^{3}$ & 0,20 & $5 \mathrm{~g}$ & 10 & 10 \\
\hline $\mathrm{E}_{2}$-Deltametrina & P.S. ${ }^{3}$ & 0,20 & $10 \mathrm{~g}$ & 20 & 0 \\
\hline $\mathrm{F}_{1}^{2}$-Fipronil & granulado & 2,0 & $1,25 \mathrm{~g}$ & 25 & 100 \\
\hline $\mathrm{F}_{2}$-Fipronil & granulado & 2,0 & $2,5 \mathrm{~g}$ & 50 & 100 \\
\hline $\mathrm{F}_{3}^{2}$-Fipronil & granulado & 2,0 & $5 \mathrm{~g}$ & 100 & 100 \\
\hline
\end{tabular}

${ }^{1}$ Tratamento padrão.

${ }^{2}$ Concentrado emulsionável.

${ }^{3}$ Pó seco.

${ }^{4}$ Em 0,5 litro de água/cupinzeiro. 
dodecacloro, mostrou-se ineficiente no controle de $C$. cumulans. Da mesma forma, a isca formicida à base de clorpirifós também demonstrou-se ineficiente nas doses testadas. Em outros trabalhos (Valério et al. 1994, Mariconi et al. 1989), com o mesmo ingrediente ativo na formulação $\mathrm{CE}$, foram registrados resultados mais eficientes, porém, com doses mais elevadas. A inclusão destas iscas formicidas deu-se em função de que a formulação granulada apresenta a vantagem de dispensar a necessidade de diluição em água. Por fim, reveleram-se igualmente ineficientes, as duas doses de deltametrina, que foi avaliado na formulação pó seco, dispensando a necessidade de água. A baixa eficiência observada confirma dados obtidos por Buainain-Alves et al. (1993). Mariconi et al. (1989), no entanto, obtiveram alta mortalidade com este produto, porém usando formulação concentrado emulsionável, e em doses 50 vezes maiores que a dose mais alta de deltametrina testada neste ensaio.

Ensaio II. O inseticida fipronil mostrou-se altamente eficiente no controle de $C$. cumulans e C. bequaerti, determinando $100 \%$ de mortalidade (Tabela 2). O mesmo não onde o produto foi colocado num ponto qualquer do ninho, a ação do produto esteve restrita, ficando na dependência da circulação dos cupins por aquele local. Mesmo a distribuição do produto via trofalaxia exigiria mais tempo. Neste caso, surege-se que a mortalidade nesta espécie venha a ser maior, caso a avaliação seja feita aguardando-se um tempo maior. Dos cinco cupinzeiros de Syntermes sp. encontrados vivos, quatro haviam sido tratados através de uma só perfuração, sugerindo que a aplicação num número maior de pontos por cupinzeiro possa resultar em maior eficiência.

Ensaio III. Em ambos os locais, o tratamento abamectina + broca cupinzeira resultou em $100 \%$ de mortalidade dos ninhos de $C$. cumulans (Tabela 3). O uso da broca cupinzeira isoladamente determinou $100 \%$ de mortalidade no local 1 e $90 \%$ no local 2 , mostrando-se eficaz como alternativa de controle, considerando as condições adotadas na presente avaliação, ou seja, completa penetração do implemento no solo e, quando necessário, mais do que uma perfuração por cupinzeiro. Um outro implemento ("demolidor de cupinzeiros") foi avaliado por

Tabela 2. Eficiência do inseticida fipronil no controle de três espécies de cupins de montículo.

\begin{tabular}{lccccc}
\hline Espécie & \multirow{2}{*}{ Formulação } & Concentração & \multicolumn{2}{c}{ Dose } & Mortalidade \\
\cline { 3 - 4 } & & i.a. $(\%)$ & Produto com. & i.a. & $(\%)$ \\
\hline Cornitermes cumulans & granulado & 2 & $5 \mathrm{~g} / \mathrm{cup}$. & $100 \mathrm{mg} / \mathrm{cup}$. & 100 \\
Cornitermes bequaerti & granulado & 2 & $5 \mathrm{~g} / \mathrm{cup}$. & $100 \mathrm{mg} / \mathrm{cup}$. & 100 \\
Syntermes $\mathrm{sp}$. & granulado & 2 & $5 \mathrm{~g} / \mathrm{m}^{2}$ & $100 \mathrm{mg} / \mathrm{m}^{2}$ & 50 \\
\hline
\end{tabular}

ocorreu, no entanto, para Syntermes sp., onde a mortalidade após 30 dias da aplicação foi de 50\%. Esta espécie não constrói ninhos com uma câmara interna à semelhança das duas outras espécies. Acredita-se que a concentração dos insetos nestas câmaras favoreça a ação do produto, até mesmo através da trofalaxia. Já para o caso de Syntermes sp.,
Ávila \& Rumiatto (1994), com resultados igualmente promissores. Entretanto, por se tratarem de implementos novos, e por serem variáveis as condições dos campos infestados (espécie de cupim, tamanho do cupinzeiro, tipo de solo, nível de umidade do solo, tipo e declividade do terreno) só com a condução de mais estudos com estes equipamentos é que 
Tabela 3. Eficiência de método mecânico ("broca cupinzeira") para o controle do cupim de montículo, Cornitermes cumulans.

\begin{tabular}{|c|c|c|c|c|c|c|}
\hline \multirow[b]{2}{*}{ Local } & \multirow[b]{2}{*}{ Tratamento } & \multirow{2}{*}{ Formulação } & \multirow{2}{*}{$\begin{array}{l}\text { Concen- } \\
\text { o tração } \\
\text { i.a. }(\%)\end{array}$} & \multicolumn{2}{|l|}{ Dose } & \multirow{2}{*}{$\begin{array}{c}\begin{array}{c}\text { Mortali- } \\
\text { dade }\end{array} \\
(\%)\end{array}$} \\
\hline & & & & Produto com. & i.a. por & \\
\hline \multirow{3}{*}{ Local 1} & & & & & & \\
\hline & Destruição mecânica & - & - & - & - & 100 \\
\hline & Abamectina + Destr. mecânica & C.E. & 1,8 & 0,2 & 3,6 & 100 \\
\hline \multirow[t]{2}{*}{ Local 2} & Destruição mecânica & - & - & - & - & 90 \\
\hline & Abamectina + Destr. mecânica & C.E. & 1,8 & 0,2 & 3,6 & 100 \\
\hline
\end{tabular}

se poderá dispor de uma avaliação mais completa sobre esta alternativa de controle.

\section{Literatura Citada}

Ávila, C.I. \& M. Rumiatto. 1994. Controle mecânico do cupim de montículo, Cornitermes cumulans (Kollar), com o implemento "demolidor de cupinzeiros", p.70-71. In: Ata e Resumos Reunião SulBrasileira de Insetos de Solo, 5, Dourados, 110p.

Buainain-Alves, C.M., J.R. Valério \& M.C.M. Oliveira. 1993. Eficiência de inseticidas no controle do cupim de montículo, Cornitermes cumulans (Kollar) (Isoptera: Termitidae) em pastagens. An. Soc. Entomol. Brasil 22: 521-525.

Cosenza, G.W. \& M.M. Carvalho. 1974. Controle e nível de dano do cupim de montículo em pastagens. Rev. Soc. Bras. Zootec. 3: 1-12.

Fernandes, P.M. \& S.B. Alves. 1991. Controle de Cornitermes cumulans (Kollar, 1832) (Isoptera, Termitidae) com Beauveria bassiana (Bals.) Vuill e Metarhizium anisopliae (Metsch) Sorok em condições de campo. An. Soc. Entomol. Brasil 20: 45-49.

Holt, J.A. \& R.J. Coventry. 1982. The effects of mound-building termites on some chemical properties of soils in Northeastern Australia. Proc.Australasian Conf. Grassl. Invert. Ecol. 3, Adelaide, p.313-319.

Mariconi, F.A.M., F.I. Geraldi, C.J. Biondo, J.L. Donatoni, A.I. Clari, F.Y. Arashiro \& A.I. Raizer. 1989. Fention e clorpirifós no combate ao cupim de monte Cornitermes cumulans (Kollar, 1832) (Isoptera, Termitidae). An. Esc. Sup. Agric.'Luiz de Queiroz" 46: 295302.

Mariconi, F.A.M., A.J. Raizer, F.Y. Arashiro, C.J. Biondo \& A.I. Clari. 1990. Combate experimental ao cupim de monte Cornitermes cumulans (Kollar, 1832) (Isoptera, Termitidae). An. Soc. Entomol. Brasil 19: 163-169.

Mariconi, F.A.M., V.B. Galan, M.T. Rocha, R.F. Maule, H.R. Passos \& R.A.A. Silva. 1994. Ensaios de combate ao cupim de monte Cornitermes cumulans (Kollar, 1832) (Isoptera, Termitidae). Sci. Agric. 51: 505-508.

Motta, R., A.J. Raizer, J.M. Silva, C.A. Sugahara, F.Y. Arashiro \& F.A.M. Mariconi. 1987. Ensaio de combate a ninhos de cupim Cornitermes cumulans (Kollar, 1832). An. Esc. Sup. Agric.’Luiz de Queiroz" 44: 1389-1395. 
Valério, J.R., C.M. Buainain-Alves \& M.C.M. Oliveira. 1994. Avaliação de inseticidas no controle do cupim de montículo, Cornitermes cumulans (Kollar) (Isoptera: Termitidae) em pastagens. An. Soc. Entomol. Brasil 23: 19-24. controle de cupins de montículo em pastagens, p.33-36. In: Ata e Resumos Reunião Sul-Brasileira de Insetos de Solo, 5, Dourados, 110p.

Valério, J.R. 1994. Ocorrência, danos e Recebido em 12/08/96. Aceito em 15/12/97. 